\title{
Extracting Analytical Nonlinear Models from Analog Circuits by Recursive Vector Fitting of Transfer Function Trajectories
}

\author{
Dimitri De Jonghe $^{\dagger}$, Dirk Deschrijver ${ }^{\ddagger}$, Tom Dhaene ${ }^{\ddagger}$ and Georges Gielen ${ }^{\dagger}$ \\ $\dagger$ : KU Leuven, ESAT-MICAS, B-3001 Heverlee, Belgium \\ $\ddagger$ : Ghent University-IBBT, Department of Information Technology, B-9000 Ghent, Belgium \\ Email: Georges.Gielen@esat.kuleuven.be
}

\begin{abstract}
This paper presents a technique for automatically extracting analytical behavioral models from the netlist of a nonlinear analog circuit. Subsequent snapshots of the internal circuit Jacobian are sampled during time-domain analysis and are then processed into Transfer Function Trajectories (TFT). The TFT data project the nonlinear dynamics of the system onto a hyperplane in the mixed state-space/frequency domain. Next Recursive Vector Fitting (RVF) algorithm is used to extract an analytical Hammerstein model out of the TFT data in an automated fashion. The resulting RVF model equations are implemented as an accurate nonlinear behavioral model in the time domain. The model is guaranteed stable by construction and can trade off complexity for accuracy. The technique is validated on a high-speed analog buffer circuit containing 70 linear and nonlinear components, showing a $7 \mathrm{X}$ speedup.
\end{abstract}

\section{INTRODUCTION}

Automated behavioral modeling of the time-domain response of analog circuits still remains a tough challenge for EDA developers. This bottleneck is mainly due to the custom nature, the increasing complexity and the nonlinear behavior of analog circuits. Although behavioral models that can simulate faster are highly wanted in industry for system verification. Recently, Model Order Reduction (MOR) techniques have successfully been extended toward nonlinear circuits by introducing trajectory piecewise (TPW) models [1], [2]. Such a model is in essence a large database of reduced-order circuit snapshots that are interpolated during model evaluation. This often requires a non-standard interaction between the SPICE simulator and the database of reduced-order snapshots.

Analytical equations can more easily be described by numerous modeling languages such as VHDL-AMS, VerilogAMS and Matlab, which improve the portability of the TPW modeling approaches. The focus of this paper is to automatically extract a set of analytical equations from a TPW approach by means of Transfer Function Trajectories [3], [4] in a more general fashion. The TFT approach enhances the transition from Modified Nodal Analysis (MNA) matrix samples toward analytical equations by transforming the samples to a mixed state-space/frequency domain [4].

In this work, the resulting TFT data are modeled using a recursive implementation of the Vector Fitting algorithm [5],

978-3-9815370-0-0/DATE13/C)2013 EDAA
[6]. The RVF technique has formerly been used to fit the frequency response of a linear circuit as a function of design parameters. It is now extended to model the time-domain response of a nonlinear circuit. The RVF model is composed of a set of fixed poles $\left\{\hat{a}_{p}\right\}$ and parameterized residues $\hat{r}_{p}($.$) .$ Afterwards, the poles and residues are converted to a system of nonlinear analytical differential equations that serve as the behavioral model. The CAFFEINE algorithm, described in [7], gives an alternative for residue regression, however because indefinite integration is required by the TFT data this cannot be automated. A schematic representation of the modeling flow from SPICE netlist to analytical differential equations is given in Fig. 1.

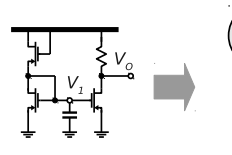

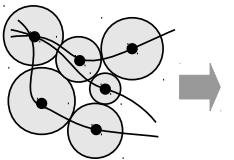

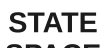

SPACE

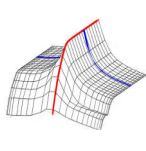

TFT
RVF

$$
\hat{f}\left(x, \frac{d}{d t} x\right)=0
$$

ANALYTICAL EQUATIONS
Fig. 1. The extraction of analytical differential equations from a SPICE netlist. Firstly, Jacobian samples are gathered throughout the state space, which in turn are transformed to a TFT hyperplane. Analytical equations are derived from this hyperplane by parametric rational approximation using the Recursive Vector Fitting algorithm.

The first step of the algorithm extracts the MNA matrix from the ELDO simulator at each time step $t_{k}$ during transient simulation, see Fig. 1. Next, the snapshots of the circuit's internal matrix are transformed to the frequency domain as TFT data which are then discretized along the frequency axis [3]. This step is described in Section II. The TFT samples are then fitted by the RVF algorithm and are translated to a Hammerstein model, see Section III. The technique is illustrated by a high-speed output buffer in section IV.

To summarize, the proposed technique enables us to:

1) extract an analytical behavioral model from a SPICE netlist

2) with high accuracy

3) in an automated fashion. 


\section{TRANSFER Function Trajectories}

Consider the state-space description of a nonlinear dynamical system of order $N$ :

$$
\frac{d}{d t} q(\mathbf{v})+i(\mathbf{v})=B \mathbf{u}, \quad \mathbf{y}=D^{T} \mathbf{v}
$$

which typically arises when modeling analog circuits using modified nodal analysis (MNA). In this paper, $\mathbf{v}=\mathbf{v}(t) \in \mathbb{R}^{N}$ is the state vector corresponding to node voltages and inductor currents in the circuit and $\mathbf{u}=\mathbf{u}(t) \in \mathbb{R}^{M_{i}}$ are inputs to the circuit. $q($.$) and i(.) \in \mathbb{R}^{N \times N}$ are matrix valued functions describing the charges and currents of nonlinear components. $B \in \mathbb{R}^{N \times M_{i}}$ is a constant incidence matrix, which maps the inputs to the internal nodes of the circuit. $D \in \mathbb{R}^{N \times M_{o}}$ is the output matrix and $\mathbf{y}=\mathbf{y}(t) \in \mathbb{R}^{M_{o}}$ the output variables.

A nonlinear system approximation for (1) is derived by approximating the Jacobians at each location $k$ in state space by a rational function in the frequency domain [3], [4]. If one is able to fix the poles $\hat{a}_{p}$ of the model over the entire state space, then the nonlinear functionality of the system approximation is fully embedded in the residues, which in turn is approximated by a rational function. The resulting approximation decouples the nonlinear functionality from the filtering function:

$$
\left\{\begin{array} { r l } 
{ 0 } & { = f ( \frac { d } { d t } \mathbf { v } , \mathbf { v } , \mathbf { u } ) } \\
{ \mathbf { y } } & { = g ( \mathbf { v } , \mathbf { u } ) }
\end{array} \Rightarrow \left\{\begin{array}{rl}
\hat{\mathbf{v}} & =\hat{f}(\mathbf{x}) \\
\frac{d}{d t} \hat{\mathbf{y}} & =\hat{A} \hat{\mathbf{y}}+\hat{B} \hat{\mathbf{v}}
\end{array}\right.\right.
$$

The hat notation denotes approximated quantities and $\mathbf{x}$ is a state estimator comprising the input $\mathbf{u}(t)$ and delayed versions of the input, as explained in more detail below.

The extraction of MNA samples in the time domain and their transform to the frequency domain is described in detail in [3], [4]. The latter results in a state-dependent transfer function $H_{l m}^{(k)}(s)$ between input $l$ and output $m$ around state $k\left(t=t_{k}\right)$ :

$$
H_{l m}^{(k)}(s)=\frac{Y_{l m}^{(k)}(s)}{U_{l}^{(k)}(s)}=D_{m}^{T}\left(\frac{\partial i}{\partial \mathbf{v}}^{(k)}+s \cdot \frac{\partial q}{\partial \mathbf{v}}^{(k)}\right)^{-1} B_{l}
$$

Here, $Y_{l m}$ is the portion of the output $Y_{m}$ due to the contribution of signal source $U_{l}$ and $H_{l m}^{(k)}(0)=D_{m}^{T}\left(\frac{\partial i}{\partial \mathbf{v}}^{(k)}\right)^{-1} B_{l}$ represents the instantaneous small-signal conductance around a trajectory or large-signal pump. The static nonlinear function $v=i^{-1}(u)$ can be reconstructed up to a constant from this set of small-signal conductance samples by indefinite integration over the input trajectory [4]. The remaining dynamical nonlinear part equals $\bar{H}_{l m}^{(k)}(s)=H_{l m}^{(k)}(s)-H_{l m}^{(k)}(0)$ [8].

The mapping of each state $k$ of the state space onto a lowdimensional state estimator $\mathbf{x}(t) \in \mathbb{R}^{q+1}$ can be constructed by adding $q$ delays $\Delta_{q_{i}}$ of the input signal $u(t)$ until each state $k$ is uniquely defined by $\mathbf{x}\left(t_{k}\right)=\mathbf{x}^{(k)}$ :

$$
k \longrightarrow \mathbf{x}(t)=\left(\mathbf{u}(t), \cdots, \mathbf{u}\left(t-\Delta_{q-1}\right)\right)
$$

A TFT approximation of the state-dependent transfer function $H_{l m}^{(k)}(s)$ with $P \ll N$ poles is given by its pole-residue form [4]:

$$
H_{l m}^{(k)}(s) \approx T_{l m}\left(\mathbf{x}^{(k)}, s\right)=\sum_{p=1}^{P} \frac{\hat{r}_{p, l m}\left(\mathbf{x}^{(k)}\right)}{s-\hat{a}_{p, l m}\left(\mathbf{x}^{(k)}\right)}
$$

A model composed of a parallel Hammerstein structure with fixed poles was proposed in [4] and is illustrated in Fig. 2. The TFT expression (5) for the parallel Hammerstein structure equals [4]:

$$
T_{l m}\left(\mathbf{x}^{(k)}, s\right)=\frac{\hat{Y}_{l m}(s)}{U_{l}(s)}=\sum_{p=1}^{P} \frac{\left.\frac{\partial}{\partial u_{l}} \hat{f}_{p, l m}(\mathbf{x})\right|_{\mathbf{x}^{(k)}}}{s-\hat{a}_{p, l m}}
$$

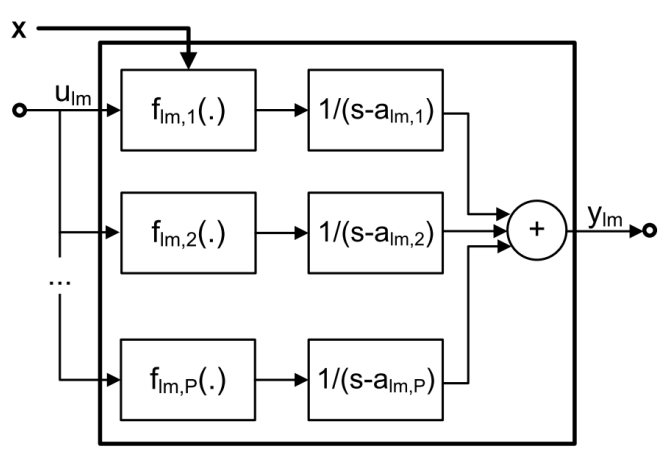

Fig. 2. Block diagram of a parallel Hammerstein TFT model.

Assuming that $\hat{r}_{p, l m}(\mathbf{x})$ can be fitted by an analytical function, the static nonlinear stages are reconstructed by integration. The corresponding time-domain representation is:

$$
\left\{\begin{array}{l}
\hat{v}_{p, l m}=\hat{f}_{p, l m, 0}+\int \hat{r}_{p, l m}(\mathbf{x}) d u_{l} \\
\frac{d}{d t} \hat{y}_{p, l m}=\hat{a}_{p, l m} \hat{y}_{p, l m}+\hat{v}_{p, l m}, \quad \hat{y}_{l m}=\sum_{p=1}^{P} \hat{y}_{p, l m}
\end{array}\right.
$$

The residue functions $\hat{r}_{p, l m}(\mathbf{x})$ are now modeled by recursively locating a set of common poles for each state-space variable by means of Recursive Vector Fitting. The advantage of this approach is that the indefinite integral in (7) exists for RVF base functions. Moreover, the integral solution is compact and only needs to be calculated once, opposed to e.g. CAFFEINE base functions. As a summary, a schematic representation of the proposed modeling chain is depicted in Fig. 3.

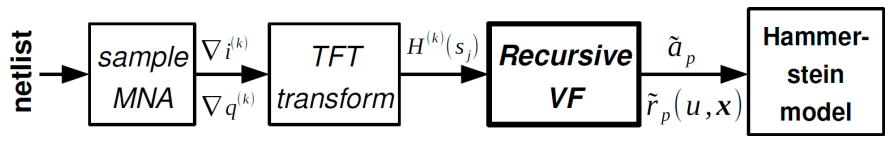

Fig. 3. Flow diagram of the TFT model extraction using RVF. The contribution of this work, RVF, is emphasized in bold. 


\section{TIME-Domain Recursive Vector FitTing}

\section{A. Vector Fitting}

The goal of the Vector Fitting algorithm is to compute a set of stable rational functions $\tilde{H}^{(k)}(s)$ from the statedependent frequency response data $\left\{s, k, H^{(k)}(s)\right\}$. The frequency response data are simply obtained by discretization of the TFT samples (3) along the frequency axis. For the sake of notation we consider Single-Input Single-Output (SISO) systems, i.e. $H_{l m}(s)=H(s)$. The extension towards MultipleInput Multiple-Output (MIMO) systems is very straightforward. A standard implementation of the relaxed Vector Fitting technique [9] returns a minimal state-space representation with $P$ stable poles $\left\{a_{p}\right\}$ and corresponding parameterized residues $\tilde{R}^{(k)}$ :

$$
\left(\begin{array}{cc}
\tilde{A} & \tilde{B} \\
\tilde{R}^{(k)} & \tilde{E}
\end{array}\right)
$$

The minimal state-space representation can be obtained by parallel connection of minimal subsystems or fractions with:

$$
\tilde{A}_{p}=a_{p} \quad \tilde{B}_{p}=1 \quad \tilde{R}_{p}^{(k)}=\tilde{r}_{p}^{(k)} \quad \tilde{E}_{p}=0
$$

provided that $a_{p}$ is real. When $a_{p}$ and $a_{p+1}$ constitute a complex pole pair (i.e. $a_{p+1}=a_{p}^{*}$ ), the corresponding statespace realization of the minimal fraction equals:

$$
\begin{array}{cc}
\tilde{A}_{p}=\left(\begin{array}{cc}
\Re\left\{\tilde{a}_{p}\right\} & \Im\left\{\tilde{a}_{p}\right\} \\
-\Im\left\{\tilde{a}_{p}\right\} & \Re\left\{\tilde{a}_{p}\right\}
\end{array}\right) & \tilde{B}_{p}=\left(\begin{array}{l}
2 \\
0
\end{array}\right) \\
\tilde{R}_{p}^{(k)}=\left(\begin{array}{cc}
\Re\left\{\tilde{r}_{p}^{(k)}\right\} & \Im\left\{\tilde{r}_{p}^{(k)}\right\}
\end{array}\right) & \tilde{E}_{p}=0
\end{array}
$$

The frequency transform of (8) in each state $k$ becomes:

$$
\tilde{H}^{(k)}(s)=\tilde{R}^{(k)} \cdot(s I-\tilde{A})^{-1} \cdot \tilde{B}
$$

The abstraction of a multiport LTI system by a parametric macromodel of the form (11) can be used for efficient design space exploration, optimization or sensitivity analysis. However, the formulation of the VF algorithm requires some slight modifications compared to [9] in order to cover the timedomain response of nonlinear dynamical systems such as the proposed parallel Hammerstein model (7). Therefore, some adaptations to the recursive VF model are now described for enabling nonlinear system modeling.

TFT expression (6) reveals that dynamical system (1) can be approximated as a parallel Hammerstein model by matching the state-dependent transfer functions $H^{(k)}(s)$ in each state $k$. When considering the Vector Fitting as a pole-residue regression tool, the state-space representation needs to be compatible with the Hammerstein structure.

It can be seen from (6) and (11) that the state-dependent residue samples $\tilde{R}^{(k)}$ coincide with the partial derivative of the nonlinear function $\hat{f}($.$) in the Hammerstein model. A block$ diagram of a single VF fraction is illustrated in the top of Fig. 4. Full compatibility of the VF model with the Hammerstein model requires the parameterized residue $\tilde{R}^{(k)}$ to be shifted towards the input. This shifted configuration is represented in the bottom part of Fig. 4.
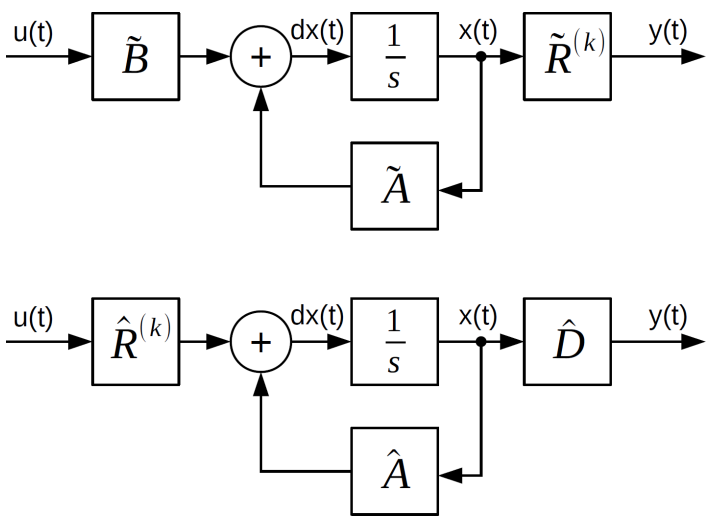

Fig. 4. Top: Block diagram of a VF fraction. Bottom: Block diagram of the transformed VF fraction, suitable for parallel Hammerstein TFT models.

A minimal state-space representation of (8) with the residue shifted towards the input (i.e. before the filtering operation) is given by:

$$
\left(\begin{array}{cc}
\hat{A} & \hat{R}^{(k)} \\
\hat{D} & 0
\end{array}\right)
$$

with a minimal fraction for real poles $a_{p}$ equal to:

$$
\hat{A}_{p}=\tilde{A}_{p}=a_{p} \quad \hat{R}_{p}^{(k)}=\tilde{R}_{p}^{(k)}=\hat{r}_{p}^{(k)} \quad \hat{D}_{p}=1
$$

Setting (8) equal to (12) at an arbitrary state $k$, the inputshifted residue for a complex conjugate pole pair can be calculated as:

$$
\hat{R}_{p}^{(k)}=\left(\begin{array}{l}
\Re\left\{\hat{r}_{p}^{(k)}\right\}+\Im\left\{\hat{r}_{p}^{(k)}\right\} \\
\Re\left\{\hat{r}_{p}^{(k)}\right\}-\Im\left\{\hat{r}_{p}^{(k)}\right\}
\end{array}\right) \quad \hat{D}_{p}=\left(\begin{array}{ll}
1 & 1
\end{array}\right)
$$

Using (12), the TFT expression (6) becomes:

$$
T\left(\mathbf{x}^{(k)}, s\right)=\hat{D} \cdot(s I-\hat{A})^{-1} \cdot \hat{R}^{(k)}
$$

The remaining parameterized residue $\hat{R}^{(k)}$ will be modeled as a partial fraction expansion in function of the state estimator $\mathbf{x}(t)$ by the Recursive Vector Fitting algorithm.

\section{B. Recursive Vector Fitting}

Similar to finding a common-pole set $\left\{\hat{a}_{p}\right\}$ for the frequency responses, a common-pole set $\left\{\hat{b}_{p}\right\}$ can be found for each of the state-dependent parameters of the parameterized residue samples. After fitting each of the $q$ state-dependent parameters in $\mathbf{x}$ as a partial fraction expansion, the dimension of the approximation problem decreases by 1 in each recursion step [6]:

$$
\hat{r}_{p}(\mathbf{x})=\hat{r}_{p}\left(u, x_{2}, \cdots, x_{q}\right)=\sum_{p 1}^{P_{1}} \frac{\hat{r}_{p 1}\left(u, x_{2}, \cdots, x_{q-1}\right)}{j x_{q}-\hat{b}_{p 1}}
$$


Regarding (6), the partial derivative of the nonlinear function blocks in the Hammerstein structure matches the residue approximation:

$$
\hat{r}_{p}(\mathbf{x})=\left.\frac{\partial}{\partial u} \hat{f}_{p}(\mathbf{x})\right|_{\mathbf{x}^{(k)}}
$$

The nonlinear function $\hat{f}_{p}(\mathbf{x})$ can be found up to a constant by indefinite integration over input $u$ :

$$
\int \hat{r}_{p}(\mathbf{x}) \cdot d u=\sum_{p_{1}}^{P_{1}} \frac{1}{j x_{q-1}-b_{p_{1}}} \cdots \sum_{p_{q}}^{P_{q}} \int \frac{\hat{r}_{\left\{p_{q}\right\}}}{j u-\hat{b}_{\left\{p_{q}\right\}}} \cdot d u
$$

with

$$
\int \frac{\hat{r}_{\left\{p_{q}\right\}}}{j u-\hat{b}_{\left\{p_{q}\right\}}} \cdot d u=j \hat{r}_{\left\{p_{q}\right\}} \cdot \log \left(j u-\hat{b}_{\left\{p_{q}\right\}}\right) .
$$

Here, $\left\{p_{q}\right\}=p_{1}, \cdots, p_{q}$ is a combination of all indices of previous iterations in the RVF model. To analytically enforce that the state-dependent base functions have a zero-phase angle, all poles $\hat{b}_{p_{q-1}}$ and $\hat{b}_{p_{q}}$ are chosen as complex pairs, which have a real part with opposite sign [10]. The remaining constant after indefinite integration can be found using the DC solution of the circuit at time $t=0$. The number of frequency and state-space poles is incremented until the error of the fitted model is below a predefined error $\varepsilon$.

As can be seen from (15) and (19), the model is fully described by the frequency and state-space poles and residues. Since both frequency and state-dependent data is fitted using the same regression engine, i.e. RVF, there is no need for choosing an appropriate regressor. Also, when using more complex regression tools for residue approximation such as CAFFEINE [4], post-calculation of the integral functions is required. A fixed regression template with existing and compact integral functions resolves this issue, as explained above in this section.

The complete time-domain RVF algorithm is listed in Algorithm 1.

The MNA matrices are extracted from ELDO SPICE and are imported into the Matlab environment. The TFT transform and RVF modelling algorithm are implemented in Matlab and the resulting RVF model was written out as a set of differential equations. The resulting system of nonlinear differential equations can be simulated inside Matlab or are further translated to the VHDL-AMS language.

\section{EXAMPle: High-SpeEd OUtPut BufFER}

The time-domain RVF algorithm is demonstrated by means of an output buffer that is used for post-amplification in an optical transimpedance amplifier. The bandwidth of the buffer equals $3 G H z$ and the DC-gain equals 2 . The buffer is implemented as a chain of 4 differential amplifiers using 27 transistors and was processed in the UMC $0.13 \mu \mathrm{m}$ CMOS technology [11]. As the buffer drives large signal inputs,
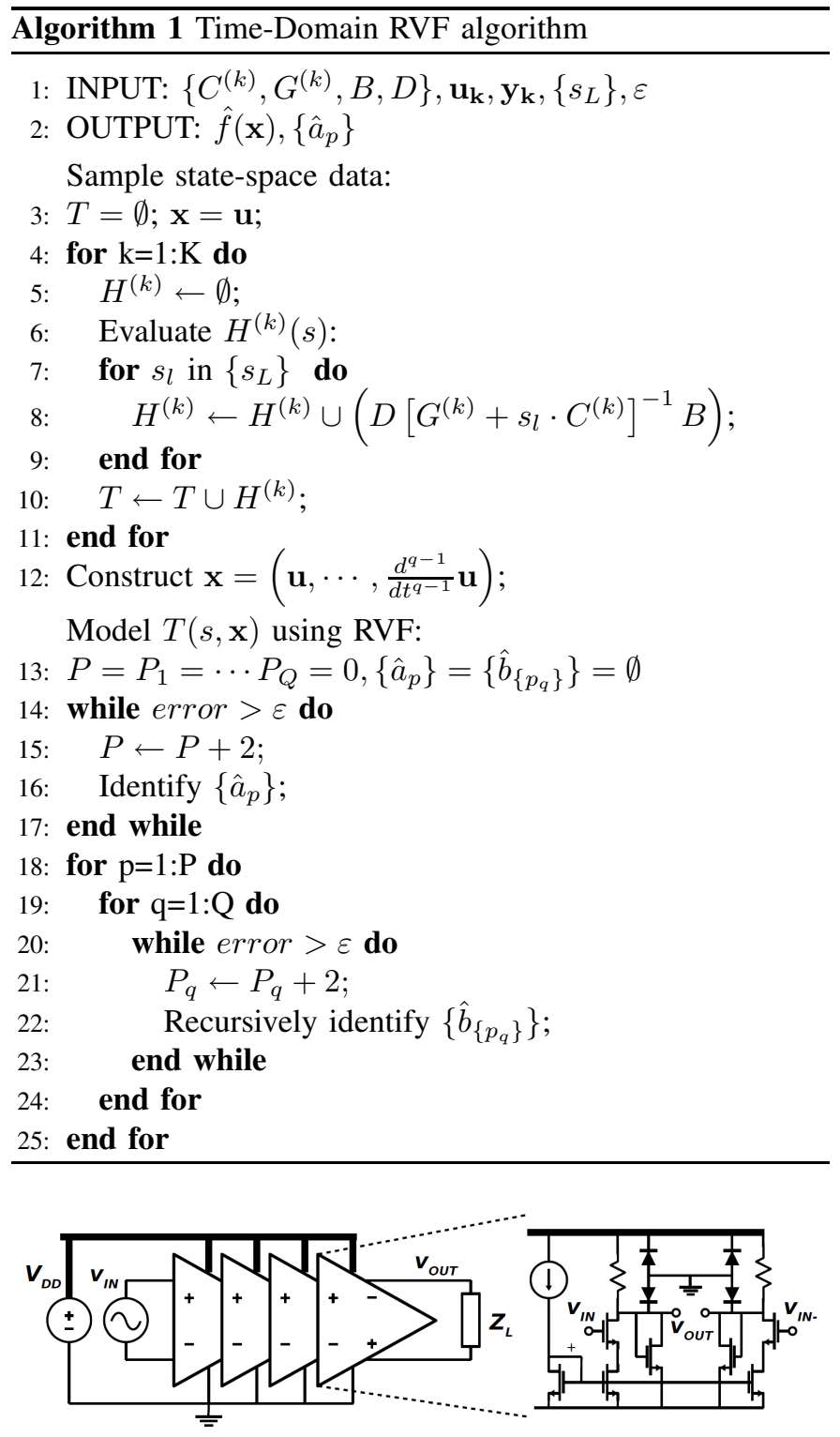

Fig. 5. Schematic representation of the high-speed output buffer.

strongly nonlinear saturation occurs for large input amplitudes. A schematic representation of the circuit is given in Fig. 5.

After simulating the circuit in SPICE with a low-frequency high-amplitude sinusoidal input for 1 period, about 100 TFT samples are collected. Only a few training points are needed for robust model extraction, as the model is based upon the internal circuit matrix. The resulting hyperplane is plotted as a function of the state-space $(\mathbf{x}=u(t))$ and frequency $s$ in Fig. 6.

After applying the RVF algorithm on the TFT dataset with error bound $\varepsilon=10^{-3}, 12$ frequency poles $\hat{a}_{p}$ and 10 statedependent poles $\hat{b}_{q}$ for each corresponding residue function $\hat{r}_{p}$ are obtained. The modeled TFT hyperplane is plotted together with the root mean square error (RMSE) in Fig. 7. The maximum RMSE was $-60 d B$ for the gain and $150^{\circ}$ for the phase occuring at high frequencies and neglegible gain 

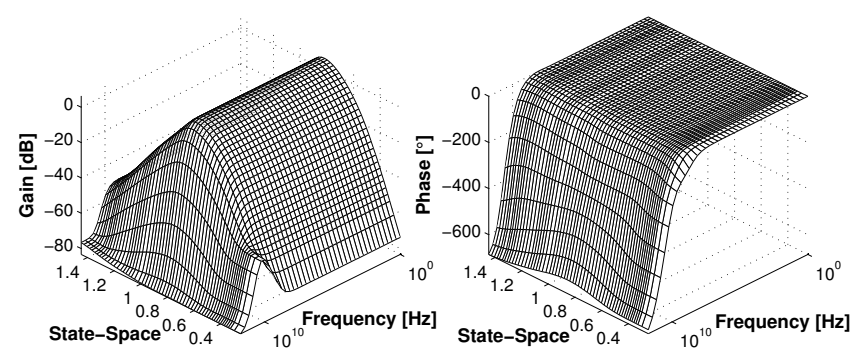

Fig. 6. TFT magnitude and phase plot of the output buffer as a function of the state space $(\mathrm{x}=u(t))$ and frequency $s$.

$(<-70 d B)$.
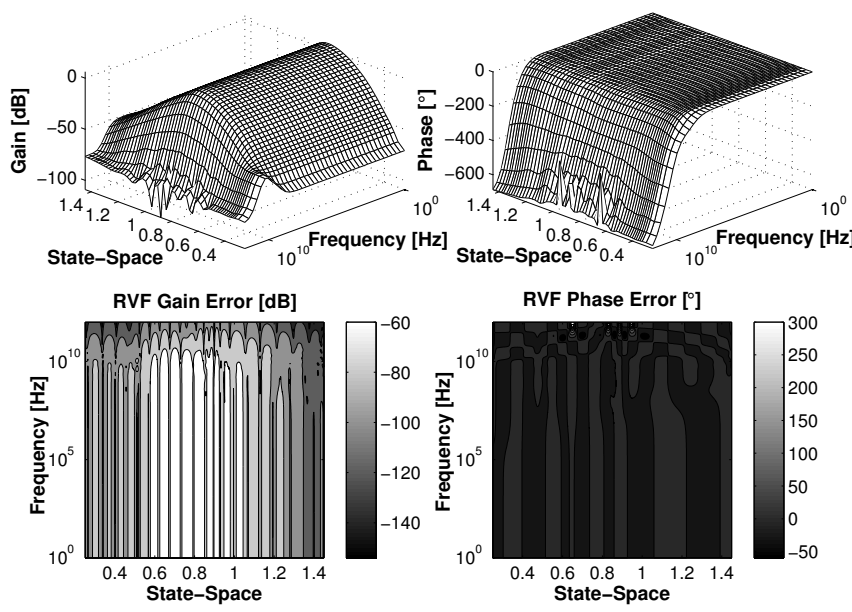

Fig. 7. Top: TFT magnitude and phase plot of the RVF model. Bottom: RMSE of both gain and phase of the RVF model compared with the TFT data.

For comparison, the same TFT data is fitted using the regular vector fitting algorithm [9] for frequency pole allocation $(P=12)$ and the CAFFEINE regression toolbox [7] is used for residue regression. The indefinite integral of the residues that are computed by CAFFEINE need to be computed manually, if they can be computed altogether. Automation is the main drawback when using CAFFEINE opposed to the RVF algorithm. The resulting error contours are displayed in Fig. 8. The maximum RMSE reaches $-20 d B$ for the gain and about $200^{\circ}-300^{\circ}$ for the phase. It can be seen that the error of the RVF model is lower and more equally distributed over the state space and frequency compared to the CAFFEINE model.

Finally a spectrally-rich bit pattern input at $2.5 G \frac{S}{s}$ is applied for testing. For comparison of the RVF approach, relatively simple base functions for the CAFFEINE algorithm are used such that the indefinite integral could be calculated manually. The results shown in Fig. 9 demonstrate a good accuracy for each of the models, with the RVF model little outperforming the CAFFEINE model. The accuracy, speed and model building time are summarized in Table I. All calculations are
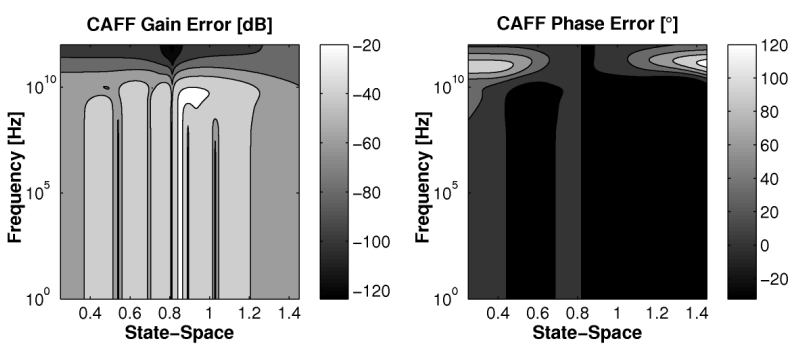

Fig. 8. RMSE of both the gain and phase of the CAFFEINE model compared to the TFT data.

performed on a $4 G H z$ dual quad-core CPU with $12 G B$ RAM.

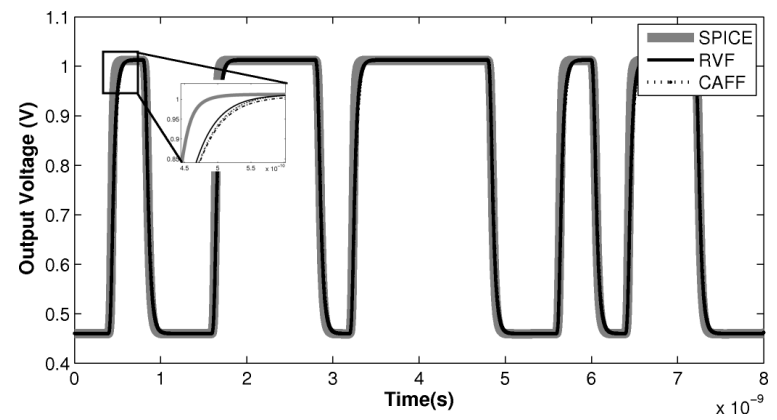

Fig. 9. Response of the different models compared to SPICE for a bit pattern input.

TABLE I

COMPARISON BETWEEN THE RVF AND CAFFEINE MODEL.

\begin{tabular}{c|l|l|l|l|l}
\hline & $\begin{array}{l}\text { Model } \\
\text { RMSE }\end{array}$ & $\begin{array}{l}\text { Time Domain } \\
\text { RMSE }\end{array}$ & $\begin{array}{l}\text { Build } \\
\text { Time }\end{array}$ & Speedup & $\begin{array}{l}\text { Fully } \\
\text { Automated }\end{array}$ \\
\hline RVF & $-62 \mathrm{~dB}$ & 0.0098 & $2 \mathrm{~min}$ & $7 X$ & YES \\
CAFF & $-22 \mathrm{~dB}$ & 0.0138 & $7 \mathrm{~min}$ & $12 X$ & NO \\
\hline
\end{tabular}

\section{CONCLUSiON}

We have presented a technique for automatically extracting analytical equations from a nonlinear analog circuit. The Recursive Vector Fitting (RVF) algorithm was used for automatically allocating frequency poles and fitting residue functions. The RVF model equations are implemented in the time domain as a Hammerstein model, which can be exported to almost any mathematical software package or behavioral description language. The model has demonstrated great accuracy for a $7 \mathrm{X}$ speedup and was verified on a high-speed analog buffer circuit.

\section{ACKNOWLEDGMENT}

The authors acknowledge the financial support of IWT/FP7 and ON Semiconductor Belgium BVBA in the frame of the GoldenGates and the SMAC projects. 


\section{REFERENCES}

[1] M. Rewienski, "A Trajectory Piecewise-linear Approach to Model Order Reduction of Nonlinear Dynamical Systems," Ph.D. dissertation, Massachusetts Institute of Technology, 2003.

[2] N. Dong, J. Roychowdhury, T. Inc, and T. Dallas, "General-Purpose Nonlinear Model-Order Reduction Using Piecewise-Polynomial Representations," IEEE Tr. on CAD, vol. 27, no. 2, pp. 249-264, 2008.

[3] D. De Jonghe and G. Gielen, "Efficient Analytical Macromodeling of Large Analog Circuits by Transfer Function Trajectories," in Proceedings of ICCAD, 2011, pp. 91-94.

[4] D. De Jonghe and G. Gielen, "Characterization of Analog Circuits Using Transfer Function Trajectories," IEEE TCAS-I: Regular Papers, vol. 59, no. 8, pp. 1796-1804, 2012.

[5] B. Gustavsen and A. Semlyen, "Rational approximation of frequency domain responses by vector fitting," IEEE Tr. on Power Delivery, vol. 14, no. 3, pp. $1052-1061$, jul 1999.

[6] T. Dhaene and D. Deschrijver, "Stable Parametric Macromodeling Using a Recursive Implementation of the Vector Fitting Algorithm," Microwave and Wireless Components Letters, IEEE, vol. 19, no. 2, pp. 59-61, 2009.

[7] T. McConaghy and G. Gielen, "Template-free symbolic performance modeling of analog circuits via canonical-form functions and genetic programming," TCAS, vol. 28, no. 8, pp. $1162-1175$, aug. 2009.

[8] E. Ngoya, N. Le Gallou, J. Nebus, H. Buret, and P. Reig, "Accurate rf and microwave system level modeling of wideband nonlinear circuits," in IEEE MTT-S MWSYM, vol. 1. IEEE, 2000, pp. 79-82.

[9] D. Deschrijver, M. Mrozowski, T. Dhaene, and D. De Zutter, "Macromodeling of multiport systems using a fast implementation of the vector fitting method," Microwave and Wireless Components Letters, IEEE, vol. 18, no. 6, pp. 383-385, 2008.

[10] D. Deschrijver and et al., "Robust parametric macromodeling using multivariate orthonormal vector fitting," $T r$ on Microwave Theory and Techniques, vol. 56, no. 7, pp. 1661 -1667, july 2008.

[11] F. Tavernier and M. Steyaert, "High-speed optical receivers with integrated photodiode in $130 \mathrm{~nm}$ cmos," Solid-State Circuits, IEEE Journal of, vol. 44, no. 10, pp. $2856-2867,2009$. 